\title{
Las Lecturas de las Cantigas de Santa María
}

Daniel Gregorio Sirem

RESUMEN.. La producción escrita de Alfonso X el sabio plantea el problema de la difusión del saber, estrechamente ligada a la práctica de la lectura y a la concepción del libro como objeto comercial y como símbolo de un poder tanto político como espiritual. Las Cantigas presentan justamente tres versiones (en verso, imagen y prosa) que permiten determinar los diferentes tipos de público que podían acceder a los textos escritos y los medios que se podían emplear para transmitir un mensaje, según el tipo de lector al que se destinaba. Las prosificaciones de las Cantigas cobran aquí una importancia frecuentemente desestimada por las investigaciones llevadas a cabo hasta ahora, pues al compararlas con las versiones rimadas o las miniaturas, se descubren los canales por los que podía divulgarse el conocimiento, al par que se matiza la oposición un tanto estereotipada entre cultura oral y cultura escrita.

Palabras clave: Alfonso X, Cantigas de Santa María, lectura, cultura, divulgación, trasmisión del conocimiento.

RÉSUMÉ. La production attribuée à Alphonse $\mathrm{X}$ de Castille pose le problème de la diffusion du savoir, en étroite relation avec la pratique de la lecture et le rôle du livre, perçu comme une marchandise ou comme un symbole du pouvoir politique et spirituel. Les Cantigas présentent trois versions (vers, image et prose) qui permettent de déterminer les différents types de public qui pouvaient accéder aux textes écrits et les moyens employés pour transmettre un message. Les versions en prose des Cantigas revêtent ici une importance toute particulière fréquemment ignorée par les chercheurs, car leur comparaison avec les versions en vers ou avec le message des miniatures permet de comprendre les voies par lesquelles la connaissance se diffusait, et nous invite à nuancer l'opposition entre la culture orale et la culture écrite.

Mots clés: Alphonse X, Cantigas de Santa María, lecture, culture, divulgation, transmisión des connaissances.

Para poder comprender la importancia sociocultural, y no sólo espiritual, de las Cantigas de Santa María, hay que determinar el público al que estaban destinadas y los diferentes modos de recepción e interpretación que éste podría tener del mensaje contenido en el manuscrito alfonsí. Ello plantea los interrogantes de la difusión del mensaje escrito, del tipo de lectura al que estaba destinado y del contexto en que dicha lectura debía realizarse. Por supuesto, aunque las respuestas sean diferentes, estos planteamientos son igualmente válidos para el conjunto de la producción alfonsina. Por ello, junto a la reflexión sobre los diversos tipos de lectura que se podían efectuar de las Cantigas, en consonancia con las diferentes circunstancias en las que se realizaban, tendremos que evocar tanto el contexto social como el objetivo político y cultural perseguido por el monarca castellano.

Todo ello nos impone tener en cuenta elementos genéricos relacionados con la producción libresca, para comprender el lugar y el papel que ocupaba el libro en el siglo XIII, así como la tipología del lector al que quedaba destinado. Ello nos permitirá mejor situar el modo de divulgación y el público apuntado por el rey sabio para el 
conjunto de sus obras y para las CSM en particular.

Es un tópico recordar que, tras la caída del imperio romano, los restos de la cultura clásica encontraron refugio en los monasterios donde se desarrolló una producción orientada hacia el estudio espiritual. En este primer período, «el códice se producía generalmente para uso de la propia comunidad, y a base de los materiales producidos y elaborados en el monasterio, quedando vinculado al mismo» ${ }^{1}$. Por esa razón, no es extraño observar que, incluso en siglos posteriores, en las miniaturas de presentación figuren monjes escribiendo. La principal función del libro en aquella época, y de la que no conseguirá deshacerse del todo en los siglos posteriores, parece haber sido «la transmisión de la palabra sagrada y el mantenimiento de las fórmulas devocionales»².

En ocasiones, sobre todo cuando el manuscrito contenía un compendio de leyes, podía transformarse en un símbolo del poder y del prestigio del soberano ${ }^{3}$. Entonces, al igual que las iglesias o los relicarios ${ }^{4}$,

-

${ }^{1}$ M. SÁNCHEZ MARIANA, Introducción al libro manuscrito, Madrid, Arco Libros, 1995, p. 14

2 I. BECERRO PITA, «La relación de las mujeres castellanas con la cultura escrita» in A. CASTILLO GÓMEZ, Libro y Lectura en la Península Ibérica y América, Consejería de Cultura y Turismo, Junta de Castilla y León, 2003, p. 15.

${ }^{3}$ G. DuBY, Le temps des cathédrales, Paris, Gallimard, 1976, p. 24 : «lorsque deux rois se rencontraient, c'était à celui qui saurait par des cadeaux plus magnifiques affirmer sa supériorité sur l'autre. Voici pourquoi les meilleurs artistes du XIe siècle se sont réunis autour des souverains tant que ceux-ci gardèrent leur puissance. L'art de ce temps est dans son principe un art aulique parce qu'il est un art sacré. Ses ateliers se relient aux cours royales. De l'éclat des différents trônes, une géographie de l'art du XIe siècle présente un reflet très fidèle».

${ }^{4}$ Ibid., p. 23: (les rois) «voulurent donc que les objets qui, sur leur ordre, étaient offerts à Dieu portassent la marque d'une certaine esthétique. Celle de l'empire- c'est-à-dire celle de Rome. (...) le soin d'orner ces offrandes majeures qu'étaient les églises, el libro materializaba el poder, tanto en el marco religioso como en el político, aunque en lo que concierne su aspecto espiritual, podía representar una «promesa de redención" ${ }^{\prime \prime}$, ya que materializaba la presencia de la palabra salvadora que anunciaba la vida en el paraíso para los justos.

Por supuesto en esta coyuntura, en la que la producción escrita quedaba cargada de un significado especial, tanto por su contenido como por quien la patrocinaba, el primer obstáculo para la difusión o posesión generalizada del libro, era el precio excesivo del mismo ${ }^{6}$, lo que significaba una imposibilidad para transformar la lectura en una actividad común y corriente. De hecho, hasta el siglo XII-XIII, la posesión del libro parecía estar condicionada por la pertenencia a cierto estamento social y al poder adquisitivo del mismo, lo que no significa que todos los miembros de la nobleza o del clero supieran leer.

Por otra parte, aunque la tasa de analfabetismo debía ser bastante alta ${ }^{7}$, debió reducirse hacia el siglo $\mathrm{XIII}^{8}$, cuando empezaron a proliferar las universidades ya fuera del ámbito monacal. En líneas generales se puede aceptar que el saber de origen

les parures de l'autel, les reliquaires et les livres illustrés où se trouvait enfermée la parole divine».

5 O. PÄCHT, L'enluminure médiévale, Paris, Macula, 1997, p.10: «Au Moyen Âge, dans le monde chrétien, le livre comme chose matérielle n'était pas un simple objet d'usage ; il avait aussi, en tant que tel, la valeur d'un signe, car il portait témoignage de la promesse du Salut, et sa valeur symbolique n'était pas moindre que celle de la Croix».

${ }^{6}$ J. VERGER, Les gens de savoir en Europe, Paris, PUF, 1997, p. 86: «le premier et principal obstacle était d'ordre économique. Le livre coûtait cher».

${ }^{7}$ R. FOSSIER, La société médiévale, Paris, Armand Colin, 1994: «la lecture livresque est l'apanage d'une minorité qui en garde jalousement le privilège».

8 J. VERGER, op. cit., p. 44: «le taux (d'alphabétisation) devait être fort bas mais on soupçonne malgré tout, à de très maigres indices, qu'il l'était peut-être moins en tout cas à partir des XIIeXIIIe siècles, qu'on ne le croit parfois». 
libresco se encontraba en manos de un grupo reducido, esencialmente compuesto por hombres que se caracterizaban por el dominio del latín y por la realización de largos estudios ${ }^{9}$, aunque ello no significase que la instrucción del resto de la población no fuera una preocupación social. No olvidemos que ya desde el siglo IX, con el renacimiento carolingio ${ }^{10}$, se desarrolló también una enseñanza orientada hacia los laicos, aunque fuertemente marcada por la cultura clerical y con contenidos muchas veces limitados a los rudimentos más básicos. Paralelamente, esta formación podía ser dispensada por tutores particulares en las familias nobles, de forma que los futuros caballeros podían recibir una formación general, aprendiendo a leer y escribir en lengua vulgar, a veces en latín, con ciertos conocimientos de orden enciclopédico ${ }^{11}$.

En uno u otro caso, esta enseñanza era impartida por el magíster, que en la iconografía medieval ${ }^{12}$ quedaba representado con el índice enhiesto, para escenificar su autoridad, y sentado en un nivel más alto que el resto del público para establecer su superioridad tanto intelectual y moral como política y social.

$\mathrm{Si}$ atendemos a estas representaciones codificadas, se podría suponer que la enseñanza se orientaba efectivamente hacia un público esencialmente masculino, aun-

\footnotetext{
${ }^{9}$ Ibid., p. 43 : «les gens de savoir n'on jamais représenté qu'une petite minorité, avant tout masculine, de la population. Leur culture était faite de discipline bien précises, d'abord difficile, ne serait-ce que par l'indispensable maîtrise préalable du latin. De longues études étaient presque toujours nécessaires, de même que la coûteuse possession de livres».

${ }^{10}$ J. PAUL, Histoire intellectuelle de l'Occident Médiéval, Paris, Armand Colin, 1998, p. 95.

11 H. MARTIN, Mentalités médiévales, Paris PUF, 1998, p. 316 y siguientes.

${ }^{12}$ AlfONSO X, Lapidario, Escorial, Monasterio, h-I15, fol. 1 .
}

que semejante idea tiene que ser matizada sobre todo en el siglo XIII, puesto que

«durante la plena Edad Media tiene lugar en Castilla y León una participación relevante en la vida pública de los miembros femeninos de la familia real, tanto por la gran dedicación guerrera de los monarcas como por el carácter todavía rudimentario de la administración, que va unido al recurso al grupo de parentesco para labores de consejo y asesoramiento. Las menciones de maestros y maestras para mujeres aparecen en torno a los años centrales del siglo XIII y se hacen más numerosas y explícitas a fines de la Edad Media. (...) al menos desde la década de 1240, la población femenina participaba en estos aprendizajes, impartido por clérigos o por maestras en la órbita monástica» ${ }^{13}$.

Y es que el siglo XIII va a representar un auténtico cambio en la difusión de la cultura y en las oportunidades para acceder a ella ${ }^{14}$.

Ello no impide que el libro siga siendo una prueba de poder. En efecto, las miniaturas de presentación del Códice de las Cantigas de Santa María, conocido como Códice de los Músicos ${ }^{15}$ o el último folio de la Biblia de san Luis, seguían manifestando la relación íntima entre la cultura, el poder político y la influencia espiritual de la Iglesia. Sin embargo, observamos ya cambios esenciales, puesto que además de los monjes aquí representados, observamos tam-

13 I. BECERRO PITA, op. cit., p. 20.

14 M. SÁNCHEZ MARIANA, op. cit., p.17: «A partir del siglo XII va a tener lugar en Europa un cambio trascendental, como es la transformación de una sociedad ruralizada y dispersa en otra agrupada en torno a unos núcleos urbanos, a consecuencia del aumento de las actividades comerciales y de la implantación de los gremios en las ciudades. (...) Como consecuencia de ello, y para satisfacer a las nuevas necesidades, la formación religiosa impartida en el monasterio habrá perdido buena parte su efectividad, y deberán aparecer nuevos sistemas de enseñanza; así surgirán los estudios generales, como respuesta a la necesidad de disponer de profesionales en leyes, medicina etc, que demanda la nueva forma de vida».

15 Alfonso X, Cantigas de Santa María, Escorial, Real Monasterio, Ms. B.I.2. 
bién la presencia de laicos que colaboran en la producción del manuscrito. Ha desaparecido el predominio indiscutible del monasterio o de la clerecía en la fabricación de los volúmenes, que por otra parte abarcan de forma abierta temas muy diversos e incluso de ocio, como pueden ser los juegos, y en los que no intervienen ya eclesiásticos.

De hecho, la proliferación de centros docentes conllevó una mayor demanda de obras escritas y por lo tanto una multiplicación de los talleres de libreros que realizaban copias de ejemplares ${ }^{16}$. Así, el libro universitario se transformó en un objeto comercial $^{17}$, un objeto de consumo relacionado principalmente con una enseñanza técnica y jurídica ${ }^{18}$.

Con el libro universitario, la actividad intelectual empezó a cambiar y, al par que cambiaban las mentalidades, manifes-

${ }^{16}$ M. SÁNCHEZ MARIANA, op. cit., p.18: «el desarrollo de los estudios trajo consigo la necesidad de disponer de nuevos textos con más urgencia de la habitual. (...) por lo que se creó en los estudios la figura del 'estacionario', cuya misión era la de conservar los ejemplares o textos aprobados por al universidad, cuidando de que la difusión de éstos se hiciese con la máxima fidelidad posible. Las copias para los estudiantes se hacían a veces de las pecia o cuadernos en que se dividía el ejemplar, de modo que pudiesen hacerse a la vez tantas copias como cuadernos tenía, ensamblando luego textos que a veces no se habían copiado en orden consecutivo. (...) Las copias podían ser realizadas por los propios estudiantes, o bien encargarse a los talleres de libreros adscritos a la universidad».

${ }^{17}$ J. VERGER, op. cit., p. 63 : «D'objets de luxe, rares, ornés, coûteux, les livres sont devenus des objets courants, de format maniable, fabriqués aux moindres frais (suppression des enluminures, multiplication des abréviations)».

${ }^{18}$ J. LE GofF, Les intellectuels au Moyen Âge, Paris, Seuil, 2000, p. 95 : «Le livre universitaire est un objet tout différent du livre du Haut Moyen Âge. Il se rattache à un contexte technique, social et économique tout nouveau. Il est l'expression d'une autre civilisation». p. 97 : «Instrument, le livre est devenu produit industriel et objet commercial». tando una curiosidad intelectual creciente, el libro fue también transformándose, o por lo menos declinándose, en varias categorías, llegando a tener una divulgación social mucho más importante. En efecto, «la progresiva secularización de las actividades intelectuales desplaza el concepto del libro como instrumento exclusivo de formación espiritual, mientras va surgiendo un progresivo interés por la cultura escrita entre las personas particulares» ${ }^{19}$.

Este interés es el que, en cierto modo, ha establecido una separación, no del todo cierta, entre cultura escrita, asociada a las elites intelectuales, y cultura oral, teóricamente más adaptada al pueblo. En realidad la división podría residir más en la forma de realizar la lectura que en el origen socioeconómico del que la realiza. La lectura pública y oral es aquella que se comparte, con la reunión de varios lectores ante un mismo volumen, o realizada por un único orador en voz alta para un público que no tiene por qué ser forzosamente analfabeto. Igualmente, la lectura personal no era sinónima de una lectura silenciosa, como la podemos entender hoy en día, puesto que el lector solitario podía frasear el texto con el fin de mejor concentrarse sobre é $\mathrm{l}^{20}$. En cualquier caso, y como lo veremos después, en los capítulos iniciales de la obra, el autor suele dirigirse a un público hipotético, lo que parece indicar que su escrito se adapta a la circunstancia de la lectura ${ }^{21}$. Es una

-

${ }^{19}$ M. SÁNCHEZ MARIANA, op. cit, p. 19.

${ }^{20} \mathrm{~J}$. COLEMAN, Public reading and the reading public in late medieval England and France, Cambridge University Press, 1996, p. 35: «Public reading and private reading should be clear enough as designations, with public carrying the sense of social, shared reading reading aloud to one or more people, not just to oneself. Private reading would mean reading to oneself, whether muttering the words or absorbing them in silence».

${ }^{21}$ Ibid., p. 101: «The author may envisage an actual audience, whose traits he will describe and whom he will address directly (sometimes in apparent jest, as in 
adaptación que tendrá que tener en cuenta no sólo el nivel intelectual del lector o del auditorio, sino también el contexto físico y la motivación, espiritual o intelectual, que genera dicha lectura. Son elementos que, precisamente, van a justificar una temática, pero que también van a modelar la apariencia física del libro, sus dimensiones, su factura y su ornamentación.

$Y$ es que el desarrollo del libro universitario no significó el abandono de la antigua producción de manuscritos miniados bajo el patrocinio de los monarcas. De hecho la asociación de la miniatura y del texto siguió demostrando el valor moral que podía tener un libro en particular ${ }^{22}$.

Es en este contexto cuando la producción libresca realizada bajo el patrocinio real pasó de ser una simple prueba de poder a una auténtica herramienta de unificación de las posesiones reales. Desde un punto de vista puramente político, el siglo $\mathrm{XIII}$ es el siglo en que empiezan a dibujarse las futuras naciones europeas; valgan como ejemplo la unificación de los reinos de Castilla y León en la península, o la anexión del Languedoc a la corona francesa tras la cruzada contra los Albigenses. Las fronteras estaban evolucionando $\mathrm{y}$ «los reyes ven frecuentemente en la cultura escrita, en un momento en que se están formando en Europa las nuevas nacionalidades y se van imponiendo las lenguas vernáculas, un instrumento de unificación y consolidación

the common reference to irate female hearers). When he speaks of his audience receiving his text, the author may occasionally refer unambiguously to private reading (...) far more often, however, he uses an apparently format-neutral read o else a hear or now hearken».

${ }^{22}$ F. LEWIS, «From image to illustration : the place of devotional images in the book of hours» in G. DUCHET-SUCHAUX, Iconographie Médiévale, image, texte, contexte, Paris, CNRS, 1990, p. 32: «Not surprisingly, it was the most frequently occurring texts which were most often illustrated, as a demonstration of their importance». de sus reinos» ${ }^{23}$. Y por supuesto, el ejemplo más evocador de esta utilización del vernáculo como herramienta de unificación sería la producción de Alfonso X.

Como heredero de la cultura erudita del siglo XII y principios del XIII, en la que el saber se había vuelto un «fin desinteresado [que] no apuntaba a proponer contra, sino paralelamente al texto revelado, un sistema coherente del mundo» ${ }^{24}$, Alfonso X asocia

«el aprendizaje de la sabiduría, los atributos del ser racional y la práctica del cristianismo. (...) La lectura, la escritura, el latín y ciertos conocimientos intelectuales resultaban imprescindibles para el control último de la maquinaria administrativa y las relaciones internacionales. (...) El libro constituye, igualmente, el instrumento de saberes especializados. Estos se corresponden, en lo esencial, con las diferentes ramas de los conocimientos universitarios, ya que, al menos en la Corona de Castilla, no se conocen apenas tratados de tipo técnico para los oficios artesanales» 25 .

Aunque no podamos aquí desarrollar todos los aspectos abarcados por Alfonso X, debemos subrayar que su obra, a grandes rasgos, podría dividirse genéricamente en cuatro campos distintos: el jurídico, el historiográfico, el lúdico/literario y el científico. Todos ellos tienen dos aspectos en común que los caracterizan.

El primero es sin duda la lengua utilizada. Toda la producción alfonsí fue redactada en castellano, como herramienta para la realización de un programa didáctico de gran envergadura, tanto en el campo de la espiritualidad y la moral ${ }^{26}$, como en el ámbito científico y político.

${ }^{23}$ M. SÁNCHEZ MARIANA, op. cit., p. 19.

${ }^{24}$ J. Verger, Les universités au Moyen Âge, Paris, PUF, 1999, p. 25.

${ }^{25}$ I. BECERRO PITA, op. cit., p.16.

${ }^{26}$ F. LAURENT, «Si douz miracle...,le Livre des miracles de Gautier de Coincy» in E. BAUMGARTNER et L. HARF-LANCNER, Seuils de l'œuvre dans le texte médiéval, 
Gracias a la lengua utilizada se puede comprender que el monarca quería dar un referente cultural común a todos sus súbditos; si el latín era la lengua de la Iglesia, era lógico que los segmentos sociales formados por las comunidades judías y musulmanas no se identificaran ni con su uso ni con las obras redactadas en él. El castellano como lengua laica podía no presentarse como un elemento extraño o ajeno a las diversas culturas de las tierras castellanas. De hecho, al venirse usando ya en obras anteriores, tanto literarias como oficiales en las chancillerías de los predecesores alfonsíes, el castellano ya tenía su propia historia, aunque aún le faltasen algunos rasgos para transformarse en una auténtica herramienta de investigación y de creación científicas. Este último paso se dio sin duda bajo Alfonso X, que al promover el desarrollo de una nueva lengua estaba intentando derribar las barreras materiales, y mentales, que impedían el desarrollo cultural, no ya entre las diferentes religiones, sino en el mismo seno de la comunidad cristiana.

En efecto, el monarca castellano buscaba

«la posibilidad de un terreno neutro y armonizador, que [hiciera] viable una vida intelectual abierta a Oriente y Occidente y [evitara] a los españoles la necesidad de aprender latín (contaban con siglos de frustración) y el árabe (era inviable), acercándose de este modo a la realización de una solución ideal a la medida de las realidades hispanas» ${ }^{27}$.

vol 2, Paris, Presses de la Sorbonne Nouvelle, 2002, p. 225 : «L'écriture est subordonnée à un projet didactique, et ce dessin affiché dès le prologue, est rappelé des préambules aux «queues", où la voix du narrateur surdétermine la fonction des Miracles par une glose moralisatrice créatrice de continuité. La récurrence de termes clés comme instruire, prendre exemple, demoustrer ainsi que l'association systématique à la rime des verbes 'réciter/esciter' informent le didactisme des récits et tissent entre eux des liens étroits».

${ }^{27}$ F. MárQUeZ VIllanUeVA, Concepto cultural alfonsí, Barcelona, Bellaterra, 2004 p.132.
Ahora bien, la utilización de una lengua vernácula, para obras que querían tener un impacto social y cultural amplio, podía reducir sensiblemente el número de lectores potenciales, limitando su área de influencia a los territorios castellanos.

Llegamos así al segundo punto en común de todas las obras alfonsinas que sería el público al que se destinaron.

Como lo subraya Márquez Villanueva, el proyecto del rey sabio no quiere identificarse con las pautas de la producción clerical clásica y se dirigía

«a la totalidad de aquellos homes qualesquier, pero no incultos, que [Alfonso X] deseaba integrasen algún día su pueblo. El esquema intelectual de que venía viviendo la cristiandad se perfilaba válido tanto en una instancia individual cualquiera como en la colectiva de la clerecía, pero no como objetivo o meta deseable para la cultura en que el rey sabio querría dejar orientado el futuro de España. Los castellanos (o al menos su rey) habían llegado al punto de juzgar conforme a sus méritos el saber clerical y no lo veían ya como un ideal de validez universal ni absoluta» ${ }^{28}$.

Ahora bien, las ideas de divulgación y de público amplio han de ser matizadas, a la vista del contexto anteriormente expuesto sobre la producción y la difusión de libros.

No se puede considerar que los manuscritos alfonsíes, por lo menos en lo que a los origínales se refiere, formaran parte de esos libros universitarios de los que hablábamos antes y por lo tanto seguían siendo objetos valiosos, cuya posesión debía estar reservada a unos pocos antes de que se realizaran hipotéticas copias más asequibles para el resto de la población. Sólo entonces podrían llagar a ser, como lo desean las Siete Partidas, auténticas obras «a servicio de Dios y a pro comunal de todos (...) porque los que lo leyesen hallasen en [ellas] todas las cosas cumplidas y ciertas para

\footnotetext{
${ }^{28}$ Ibid., p. 138.
} 
aprovecharse de ellas» ${ }^{29}$. La idea de una utilización práctica del conocimiento adquirido queda entonces ligada a una mayor facilidad en el aprendizaje. El rey sabio ordena la redacción de libros para que «los que esto quisiesen aprender lo pudiesen más de ligero saber, non tan solamente por entendimiento más aún por vista» ${ }^{30}$. Aunque también es cierto que la difusión del saber no significaba poner al alcance de cualquiera cualquier tipo de conocimiento, y de hecho el prólogo del Lapidario recuerda que

«este libro es muy noble et muy preciado. Et qui del se quisiere aprovechar conviene que pare mientes en tres cosas. La primera, que sea sabidor de astronomía, porque sepa conocer las estrellas, en cual estado están, et en qual sazón viene mayor vertud a las piedras dellas segund la vertud que reciben de Dios. La segunda cosa es que sepan conocer las piedras et las colores et las faiciones dellas et otrossi que estrema la contrafecha del natural et departir otrossi las que natural miente se semeian en uno, conociendo las por peso et por dureza et por las otras señales por que se pueden conocer a ombe que fuere entendudo en este saber. La tercer cosa es que sea sabidor del arte de física que iaze mucho del la encerrada en la vertud delas piedras segund en este libro se muestra. Et que sepa dellas obrar así como en el manda. Et que sea de buen seso por que se sepa ayudar de las cosas que fazen pro et se guarde de las que tienen daño» ${ }^{31}$.

Todo ello nos induce a pensar que el alcance de estas creaciones, en su versión original, debió ser limitado en un principio, por no corresponder a una producción de masa y estar destinadas a un público con cierto bagaje cultural. De hecho, aunque por razones tanto políticas como técnicas, muchas obras alfonsinas, como las Siete Partidas o las de orden historiográfico, no

\footnotetext{
${ }^{29}$ Alfonso X, Siete Partidas, Biblioteca Nacional de Madrid, I-766, P1, T1, proemio, fol. 3r.

${ }^{30}$ AlfONSO X, Libros del saber de astronomía, Biblioteca Universitaria Complutense de Madrid, 156, fol. $1 \mathrm{v}$.

${ }^{31}$ AlFONSO X, Lapidario, Monasterio de El Escorial, h-I-15, fol. $1 \mathrm{v}$.
}

empezaron realmente a tener un verdadero impacto intelectual hasta años más tarde, cuando fueron acabadas y remozadas por los sucesores del rey sabio ${ }^{32}$. Las obras de carácter más científico tuvieron un destino un tanto diferente puesto que «se transmitieron libremente entre centros de estudio o entre especialistas» ${ }^{33}$, como ocurrió con las Tablas Alfonsíes que representaban uno de los fundamentos esenciales de la astronomía medieval occidental hasta el siglo XVI y el abandono del sistema ptolemaico por el de Copérnico.

Capítulo aparte merecen las Cantigas de Santa María ${ }^{34}$ puesto que representan la obra más íntima del rey sabio, al no haber sido terminadas o alteradas por otros monarcas. Estaban igualmente destinadas a participar en ese proyecto alfonsí de divulgación del saber y de la cultura, aunque de forma muy distinta a la de las demás obras.

En primer lugar, el patrocinio y la posesión de una obra como las Cantigas demostraban tanto la personalidad piadosa del monarca como su poder político. A ello se añade un valor intelectual indudable. Alfonso $\mathrm{X}$ tenía que proponer a sus súbditos un soporte para la reflexión espiritual, al igual que lo había hecho en el campo legislativo o científico, si quería realmente abarcar todas las facetas del conocimiento humano. Y es por ello que las CSM, además de ofrecernos la oportunidad de percibir ciertas facetas de la vida cotidiana del siglo XIII, «evidencian el afán enciclopédico del monarca, puesto que a la belleza poética se une la armonía musical y plástica, esta

${ }^{32}$ H. SALVADOR MARTínEZ, Alfonso X, el sabio, una biografía, Madrid, Polifemo, 2003, p.269.

${ }^{33}$ M. SCHAFFER, «Los códices de las Cantigas de Santa María: su problemática» in J. MONTOYA y A. DOMÍNGUEZ RODRÍGUEZ, El scriptorium alfonsí: de los libros de Astrología a las Cantigas de Santa María, U Complutense, Madrid, 1999, p 133.

${ }^{34}$ AlfONSO X, Cantigas de Santa María, Escorial, Real Monasterio, Ms. T.I.1. 
última representada por las innumerables miniaturas que ilustran los códices» ${ }^{35}$. Por supuesto la noción de belleza ha de ser entendida según los cánones medievales.

Retendremos por una parte que el ideal de belleza, heredado de la Antigüedad, aunaba tanto la belleza física como moral $^{36}$, de ahí que se pudiera también considerar como una manifestación de la voluntad o de la perfección divina ${ }^{37}$. En el siglo XIII, la relación armónica de las partes constitutivas de un todo como fundamento esencial de una obra estética abarcaba tanto los elementos físicos como la presencia de la luz como valor místico de esencia puramente divina ${ }^{38}$, que podía manifestarse en los objetos físicos y en las composiciones literarias ${ }^{39}$.

${ }^{35}$ F. Mundi y A. SAÍZ, Las prosificaciones de las Cantigas de Santa María, Barccelona, PPU, 1987, p. 23.

${ }^{36}$ E. DE BRUYNE, Études d'esthétique médiévale, 2 vol, Paris, Albin Michel, 1998, vol I, p. 476: «La beauté formelle va souvent de pair avec la beauté physique et morale : l'art antique n'est pas une représentation du réel, mais l'expression d'un idéal plus beau que la nature».

${ }^{37}$ Ibid., vol II, p. 6-7 : «L'esthétique du XIIIe siècles est donc issue de problèmes théologiques et elle présente un caractère profondément religieux : la beauté des choses est fonction de celle du Créateur et l'attitude de que l'homme doit prendre vis-à-vis de la beauté et des plaisirs qui en découlent, et commandée par la morale générale du christianisme».

${ }^{38}$ V. NiETO ALCAIDE, La luz y el símbolo visual, Madrid Cátedra, 1989, p. 39 y siguientes.

${ }^{39}$ E. DE BRUYNE, op. cit., vol II, p. 9 : «L'esthétique du XIIIe siècle se développe dans un climat particulier, celui d'une mystique de la lumière. Certes, nous ne disons pas que l'idée de proportion disparaît : elle ne perd rien e son importance fondamentale. Mais alors que le XIIe siècle insiste particulièrement sur la composition c'est-à-dire sur la beauté de la composition musicale ou littéraire, de la composition architecturale ou plastique, de la composition du corps humain, le XIIIle siècle attache une importance considérable à tout ce qui est clarté, lumière, splendeur. Les systèmes esthétiques enfermés dans les Somme immenses ou dans les opuscules plus modestes ne prennent toute leur signification qu'en fonction d'un véritable esthétique diffuse de la Lumière. Celle-ci se manifeste sous
La organización física de los poemas a lo largo de las CSM, así como la unión de la imagen, de la música y del texto demuestran que la colección de milagros aspiraba a unificar todos estos elementos para ser lo más perfecta posible, lo que puede explicar las sucesivas versiones claramente identifi$\operatorname{cadas}^{40}$, cada vez aumentadas con nuevas narraciones y diferentes elementos estéticos, siendo el Códice Rico, con sus más de cuatrocientos poemas, la versión más extensa y acabada.

Se trata de una producción en galaico-portugués, con anotaciones musicales que incorpora en su última versión miniaturas que van ilustrando la narración correspondiente. Son, como lo han indicado casi todos los investigadores que se han ocupado de estos poemas marianos ${ }^{41}$, un

trois formes principales: une forme littéraire, une forme mystique, une forme physique ; Ne croyons pas qu'il y ait influence directe d'une de ces formes sur l'autre».

${ }^{40}$ W. MetTMAnN, «Algunas observaciones sobre la génesis de la colección de las Cantigas de Santa María y sobre el problema del autor» y J. MONTOYA MARTíNEZ «Algunas precisiones acerca de las Cantigas de Santa María» En J. KATS \& J. KelleR,_Studies on the Cantigas de Santa María, Madison, Hispanic Seminary of Medieval Studies, 1987.

${ }^{41}$ Como ejemplo, en absoluto restrictivo, podemos referirnos a M. SCHAFFER (Los códices de la s CSM su problemática, p.128: «ya que los códices marianos son los más complejos de los alfonsíes, por incorporar programas complejos de música y miniatura con texto en verso, no han sido investigados sistemáticamente.) a J. T. SNOW (Alfonso X y las cantigas, p. 160: «El manuscrito que se elaboraba como, tal vez edición definitiva que se designa hoy como Códice Rico (...) contiene texto, música y miniaturas», a F. MÁRQUEZ VILLANUEVA, op. cit, p.114: «Sus 420 piezas no sólo constituyen uno de los grandes fresco abarcadores de la vida medieval en toda su riqueza de luces y sombras, sino aparte de su siempre encarecido valor como testimonio de piedad religiosa, las CSM son también un ámbito poético de gran novedad y encanto» y a H. SALVADOR MARTíNEZ, op. cit., p.237: «las CSM con sus 420 composiciones y 2400 miniaturas constituyen el cancionero mariano más rico de toda la Edad Media y el repertorio musical más importante de Europa por lo que se refiere a la lírica medieval. Las CSM se conser- 
auténtico monumento literario compuesto esencialmente de tres partes complementarias: verso, imagen y música.

La estructura general de la obra asocia una narración de milagro en verso, destinada a ser cantada, y una serie de seis miniaturas que explican o representan el conjunto de la narración. La relación entre texto e imagen queda subrayada por la presencia de esa filacteria en la parte superior de cada miniatura y que resume la escena representada. Dicha estructura sólo cambia cada cinco poemas, con composiciones más largas acompañadas por doce miniaturas a doble página; las cantigas decenales, pese a quedar acompañadas por seis miniaturas, no son narraciones de milagros sino auténticos poemas de loor, expresiones de la devoción regia ante la Virgen. Veremos más adelante la importancia de esta estructura para la recepción de la obra por parte del público, pero por el momento, podemos observar que el Códice Rico presenta ya todos los elementos antes evocados para la consecución de una obra bella, física y moralmente hablando. Nos presenta una relación armoniosa de sus componentes, intenta atrapar y expresar la luz del mundo con sus miniaturas o el equilibrio de la creación à través de la parte musical. A todo ello viene a añadirse une elemento suplementario que, sin embargo, suele quedar en el olvido. Se trata de la versión en prosa castellana de los veinticinco primeros poemas, y que se encuentran en la parte inferior de los folios. A nuestro entender estas versiones son las que mejor permiten comprender las personalidades de los diferentes públicos a los que se destinaba la obra en su conjunto.

Empecemos por recordar que los primeros milagros redactados en latín por

van en cuatro espléndidos manuscrito compuestos con toda probabilidad para la cámara regia y para uso personal del Rey y su corte». autores como Gautier de Cluny (De Miraculis Beatae Mariae Virginis), Venancio Fortunato (Ave Maris Stella), Pothon (Liber de Miraculis Sanctae Dei Genitricis Mariae), o Vincent de Beauvais (Mariale Magnum) iban dirigidos a un público muy particular, noble o eclesiástico, que podía deleitarse y buscar una enseñanza moral con una lectura personal o escuchando una lectura en voz alta por otra persona. La traducción en lengua vernácula de tales obras, realizada por autores como Adgar, Gautier de Coincy y Gonzalo de Berceo, nos muestra que el libro en latín no representaba una obra inconmovible sino una producción que podía seguir evolucionando en el tiempo, sobre todo gracias a las traducciones y a las diferentes versiones que de una misma obra se iban realizando ${ }^{42}$. Por otra parte, estas versiones en vernáculo, no alteraron significativamente las características del público al que iban destinados los escritos. En efecto, el epílogo de los Miracles de la Sainte Vierge de Coincy hace especial mención a las «damoiselles, aus cloistrières de Nostre Dame de Soissons ${ }^{43}$, aunque no excluye tampoco a los «Roys et Roynes, Dus, Duchesses (...) Abbé, Abbesses, moines et clers» ${ }^{44}$ que deben encontrar la inspiración necesaria en estos poemas. De la misma forma, la introducción de los Milagros de Nuestra Señora de Berceo indica el comportamiento que el poeta ha de tener ante un público cuya sensibilidad artística le debería permitir comprender el mensaje implícito en las narraciones y no detenerse sólo en la forma

-

${ }^{42}$ F. LAURENT, op. cit., p. 224 : «Le livre latin n'est donc pas perçu comme un monument immuable, donné de tout éternité et pour toujours, mais il est pensé dans le temps, et il n'existe que grâce au travail de traduction qui lui redonne une nouvelle vie».

${ }^{43}$ G. DE COINCY, Miracles de Nostre Dame, Genève, Slatkine 1972, « De la chasteté aux nonnains »

${ }^{44}$ Ibid., Épilogue, v 112-1116. 
poética de las mismas ${ }^{45}$. Este comportamiento recuerda en parte la definición dada por Alfonso X, en lo que concierne la creación poética, según la cual «trobar é cousa en que jaz entendimento ${ }^{46}$.

El rey sabio parece pues escribir sus poemas para un público culto, seguramente inclinado a poseer libros o por lo menos a manejarlos en el contexto general que evocábamos al iniciar esta reflexión. Parece evidente que las CSM iban dirigidas en un principio a esos lectores de seso, evocados en el prólogo del Lapidario. Así, la redacción de los milagros, bajo forma de narración en prosa, podría exponer todo un proyecto diferenciado de divulgación, puesto que cada estilo y forma de composición tiene su propio contexto de desarrollo y necesita una sensibilidad artística específica para ser comprendido $^{47}$. Recordemos que, como ya lo indicara Carlos Alvar,

«la poesía lírica medieval se caracteriza por la coexistencia de tres corrientes perfectamente definidas $y$, en principio, delimitadas con toda claridad: la lírica de tipo tradicional, en lengua vernácula; la lírica culta, escrita en latín; y la lírica cortés, culta, pero escrita en lengua vulgar. Cada una de estas corrientes tiene su propio público y, también, sus propios intérpretes y cauces, difundiéndose de forma independiente entre sí. No obstante, hay veces en que convergen $y$ llegan a superponerse las tres tendencias» ${ }^{48}$.

Ello no puede hacernos olvidar que, pese a presentarse como poemas de loor $y$

-

${ }^{45}$ G. DE BERCEO, Milagros de Nuestra Señora, Madrid, Espasa Calpe 1991; Prólogo, § 16: «prendamos lo de dentro, lo de fuera dessemos».

${ }^{46}$ Alfonso X, Cantigas de Santa María, Prólogo B, v. 1-2, edición de Walter METTMAN, Madrid Castalia, 3 vol, 1988.

${ }^{47}$ J. COLEMAN, op. cit., p. 27: «it is undeniable that, at their poles, orality and literacy describe distinct forms of literacy experience. Bards composing extemporaneously in crowded mead halls and authors writing in studies for armchair readers operate in radically different environments».

${ }^{48}$ C. Alvar, Poesía Lírica Medieval, Madrid, Taurus, 1987, p.15. como ofrenda a la Virgen, las CSM fueron redactadas para ser transmitidas a los demás, no debían ser una creación que quedara ignorada por el resto de la cristiandad.

De hecho, tanto en el Prólogo B como en la cantiga $401^{49}$, Alfonso X pide la intercesión de la Virgen en su favor para obtener el perdón de sus pecados. El monarca proclama entonces que semejante protección por parte de María tendría como consecuencia más directa que los otros poetas se pusieran a su servicio ${ }^{50}$. Ello indica que las CSM debían transformarse en una referencia para los demás autores, un modelo a seguir para salvar sus propias almas. Por supuesto este papel de ejemplaridad no es propio de las CSM puesto que Berceo anuncia en su introducción que su mensaje ha de ser considerado como bueno en ver$\operatorname{dad}^{51}$, mientras que Coincy amenaza con grandes sufrimientos a aquellos que habiendo leído sus poemas no se pongan al servicio de Dios y de Su madre ${ }^{52}$.

Todas estas composiciones se destinaban a la educación religiosa y a la salvaguarda del alma del público presente. Ahora bien, además del evidente valor moral que encierran las CSM por el tema que abordan, el escrito alfonsí posee un valor suplementario al de las demás composiciones y que estriba en la mera presencia física del códice. En efecto, en el segundo testa-

\footnotetext{
${ }^{49}$ Tanto para las cantigas en verso como para las prosificaciones correspondientes utilizaremos la edición de Walter METTMAN.

${ }^{50}$ Prólogo B, v. 43-44: « queno souber, // por ela mais de grado trobará $»$.

${ }^{51}$ G. DE BERCEO, Milagros..., introducción §1: «tenédeslo en cabo, por bueno verament ».

${ }^{52}$ G. DE COINCY, Miracles ..., épilogue, 681, v. 1-7: «qui ces miracles a leuz, bien est chaitis, bien durfeuz (...), se soupris n'est de bien servir la douce Dame, qui tant homme et tante fame en paradis sachie ». «quiconque aura lu ces miracles, sera bien châtié et bien malheureux (...) s'il ne se voue pas au service de la douce Dame qui a accordé le paradis à tant d'hommes et tant de femmes »
} 
mento del rey sabio, otorgado en 1284, el monarca deseaba que «todos los libros de los Cantares de loor de Sancta María sean todos en aquella iglesia do [su] cuerpo se enterrare, e que las fagan cantar en las fiestas de Sancta María» ${ }^{53}$

En líneas generales, la poesía mariana se destinaba a la edificación espiritual del público y rebasaba así su simple papel de creación literaria para transformarse en una obra docente además de deleite ${ }^{54}$. Pero, puesto que las CSM debían quedar expuestas en la capilla real y ser leídas en momentos específicos del año litúrgico, parece evidente que el público que podía tener acceso directo a esta obra y contemplar las miniaturas, resultaba muy limitado. La auténtica divulgación de las Cantigas sólo podía realizarse por la transmisión oral, en voz de diferentes trovadores que podían abandonar la corte alfonsí para cantar y repetir en otros lugares las creaciones originales $^{55}$. Y es que, como obra poética, las CSM parecen ser las herederas de dos corrientes particulares. A la influencia religiosa habría que añadir la puramente trovadoresca que en el ámbito peninsular se desarrolló con fuerza en el siglo XIII gracias a

${ }^{53}$ H. SAlvadOR, op. cit., Apéndice IX, codicilo al testamento, p 618.

${ }^{54}$ E. DE BRUYNE, op. cit., vol I, p. 691 : «La poésie a un double but : instruire et délecter. Par sa fin sérieuse elle relève du savoir universel, c'est-à-dire de la philosophie, par sa fin délectable, du jeu, c'est-à-dire de la jonglerie : elle enseigne en jouant».

${ }^{55}$ Ibid., vol I, p. 488 : «Au fond le Moyen-Âge connaît trois types de musiciens - que souvent il appelle indifféremment musici ou cantores. Le musicien savant est le mathématicien qui ne doit ni composer ni chanter (...) Le musicien parfait est à la fois savant compositeur, chantre, il applique sa science abstraite à la réalisation de l'harmonie dans les sons et la voix (...) le troisième type de musicien, au contraire, ne sait rien de la théorie spéculative : il ne fiat que créer (operari) en se servant de ses mains ou de sa voix».
Alfonso X en Castilla y a don Denis en Portugal $^{56}$.

Las investigaciones realizadas insisten en la más que probable participación de poetas profesionales y en la influencia de algunos trovadores provenzales sobre $\mathrm{Al}$ fonso $X^{57}$. Así, la estancia de Guiraut de Riquier, de Airas Núñez y de Cadenet en la corte del rey castellano podría explicar las similitudes entre diversos poemas de loor y las creaciones personales de estos poetas. Los temas del arrepentimiento y de la muerte, presentes ya en la obra de Guiraut de Riquier (En tôt quand qu'ien saupes), la crítica de la vida mundana y de las vanidades humanas realizada por Guiraut de Borneh (Be vog e conosc e saj), se encuentran también presentes en las cantigas de loor $\left(n^{\circ} 130,170,190,240\right)$. Estas cantigas que se refieren a las angustias y a las esperanzas de los hombres de aquella época son las que permiten concebir las CSM como « una motivación personal y una contribución organizada a la poesía mariana del siglo treces ${ }^{58}$.

\footnotetext{
${ }^{56}$ C. Alvar, op. cit., p.54-55: «Los periodos alfonsí y dionisíaco constituyen la época clásica de la poesía cortés gallego-portuguesa, y están marcados por la personalidad de los dos reyes, protectores de las letras y poetas ellos mismos: Alfonso X (rey desde 1252 a 1284) y don Denís (rey desde 1279 a 1325). La abundancia de poetas que florecieron en Castilla y Portugal durante el reinado de estos dos monarcas, hace muy difícil establecer una cronología; esta dificultad se ve agravada por la escasez de datos, el continuo movimiento de algunos nobles y el elevado número de poetas que sólo compusieron cantigas de amor o de amigo, carentes de cualquier referencia al mundo que les rodeaba y, por tanto, imposibles de fechar».

${ }^{57}$ H. SALVADOR, op. cit. p.243: «De muchos de estos poetas gallego-portugueses y provenzales Alfonso debió recibir ayuda, tanto en la recolección de materiales como en la técnica de la composición y la expresión poética».

${ }^{58}$ J. T. SNOW, «The central role of troubadour persona of Alfonso X in the Cantigas de Santa María ", in Bulletin of Hispanic Studies, Liverpool, Liverpool University Press 1979, p. 305: «a more personally moti-
} 
La colaboración de estos poetas, y sobre todo su origen internacional, favorecería una posible divulgación de los poemas alfonsíes, aunque siempre en un ambiente cortés, pero lo más importante es que esta difusión en las cortes o núcleos culturales occidentales rompía la armonía existente entre imagen y texto. La divulgación de estos poemas gracias a la sola transmisión oral significaría una ruptura o alteración del objetivo inicialmente apuntado por las CSM, consideradas como un todo. No olvidemos que la imagen es el medio por el cual la devoción parece mejor plasmarse al permitir una mejor exaltación de los sentimientos ${ }^{59}$.

Nos encontramos pues por el momento con un doble tipo de lectura, el primero puramente artístico con tintes religiosos efectuado por los eventuales trovadores fuera de la capilla real, y el segundo inscrito en un ambiente piadoso dentro del marco religioso y litúrgico, donde pueden cantarse los milagros, pero donde también pueden contemplarse bajo forma de historia miniada. En este segundo tipo de lectura, las imágenes piadosas, sobre todo cuando evocan la pasión de Cristo, contienen una fuerte carga emocional ${ }^{60}$ que alimenta las representaciones mentales de cada espectador ${ }^{61}$. Esta contemplación, por definición interior

vated and organized contribution to the Marian poetry of the thirteenth century».

${ }^{59}$ F. LEWIS, op. cit., p. 30: «the texts provide the words through which devotion speaks, the images through which the objects if devotion are visualised, and thus they play an affective as well as reflective role».

${ }^{60} \mathrm{H}$. BeLtiNG, L'image et son public au Moyen Âge, Paris, Gérard Monfort, 1998, p .99: «D'une manière générale, l'image de dévotion a été interprétée en termes psychologiques, c'est-à-dire comme une image sollicitant l'affectivité du spectateur et permettant l'engagement $d^{\prime}$ un dialogue personnel avec la figure représentée».

${ }^{61}$ H. MARTIN, op. cit., p. 122 : «En somme, les images renvoient à d'autres représentations, mentales celles-là». y solitaria puede introducir la idea de una lectura más personal y no pública o colecti$\mathrm{va}^{62}$.

Si es cierto que en la Edad Media la lectura de un texto no solía ser un acto aislado ${ }^{63}$, la contemplación de un mensaje iconográfico, aunque se pudiera realizar rodeado por una muchedumbre, debía desembocar en una interpretación mucho más personal de la historia representada, puesto que implicaba la sensibilidad propia de cada espectador ${ }^{64}$. El caso de las CSM, cuyo manuscrito debía quedarse en un ambiente esencialmente culto, demuestra que las imágenes rebasan el simple marco de la decoración o de la adoración ${ }^{65}$, para

-

${ }^{62}$ J. COlEman, op. cit., p. 35: «Public reading and private reading should be clear enough as designations, with public carrying the sense of social, shared reading -reading aloud to one or more people, not just to oneself. Private reading would mean reading to oneself, whether muttering the words or absorbing them in silence. An alternate term for public reading is prelection».

${ }^{63}$ R. JACOB, Images de la justice, Paris, Le Léopard d'or, 1994, vol II, p. 178. «l'enluminure de la Bible, de la littérature hagiographique, des chroniques, des romans de chevalerie, avait d'abord pour fonction de rendre palpable la matière du récit. $D^{\prime}$ en entretenir la mémoire. Ordinairement la lecture n'était pas au Moyen Age un acte solitaire accompli dans l'intimité. Elle se faisait en commun et à haute voix»

${ }^{64}$ H. BELTING, op. cit., p.60 : «L'imago réinterprétée et l'histoire transformée constituent toutes deux, pour ainsi dire, des stations propices ç une médiation prolongée. (...) Dès le XIIIle siècle le morcellement de la suite narrative en haltes pour la contemplation étaie déjà un principe d'écriture des Mediationes. Dès lors que le récit de la Bible est apparu comme fournissant un matériau brut pou l'élaboration de ce que les fidèles souhaitaient contempler, l'évolution ne pouvait pas rester sans conséquence dans le domaine de la représentation visuelle». P.99: «D'une manière générale, l'image de dévotion a été interprétée en termes psychologiques, c'est-à-dire comme une image sollicitant l'affectivité du spectateur et permettant l'engagement d'un dialogue personnel avec la figure représentée».

${ }^{65}$ E. DE BRUYNE, op. cit., vol I, p. 264 : «en 1025 le Concile $\mathrm{d}^{\prime}$ Arras résume très bien le premier argument anti-iconoclastique : l'art est une enseignement. Ce que sont les lettres pour les savants, les images le sont pour les illettrés». 
facilitar la exaltación religiosa; al quedar presentadas esencialmente a miembros de la corte, las miniaturas no debían tener como primer objetivo la explicación del texto o el de facilitar la comprensión de la historia a un público analfabeto, sino que tenían que favorecer y fortalecer la devoción mariana.

Se plantea entonces el problema de las prosificaciones.

Basándonos en el trabajo realizado por Francisco Mundi y Anabel Sáenz, destacaremos en un principio que

«la letra con que están escritas las prosificaciones es mucho más pequeña que la de las cantigas en gallego, que aparecen en los mismo folios. Esto también puede permitirnos pensar que los traductores sólo intentaban aclarar el texto gallego, pero nos resistimos a considerar que estas prosificaciones sean sólo glosas porque, a menudo, aportan datos que en su modelo gallego no aparecen» ${ }^{66}$.

Por otra parte, si consideramos que la prosificación de la cantiga veinticinco quedó inacabada, es evidente que el proyecto de verter las Cantigas al castellano se estancó en un estado muy primitivo de realización. Ello nos permite abundar en la idea de que «las prosificaciones debieron iniciarse a finales del reinado de Alfonso $X_{\text {» }}$ lo que no impide que pudieran tener «un propósito de divulgación generalizada que no tenían las Cantigas gallegas» ${ }^{67}$.

Las prosificaciones no son, ni mucho menos, una mera traducción o versión más

S. RINGBOM, Les images de dévotion, Paris, Gérard Monfort, 1995, p.46: «le support procuré par le texte était aussi utile qu'il y paraît. Dans un livre, une illustration n'est pas tenue d'être parfaitement explicite ; le lecteur suit le texte et images plus ou moins simultanément, l'image rehaussant le texte et le texte commentant l'image. Dans d'autres domaines artistiques, le spectateur peut également être aidé par des légendes à comprendre le sujet d'une représentation».

\footnotetext{
${ }^{66}$ F. MUNDI y A. SAíz, op. cit., p.38.

${ }^{67}$ Ibid., p.37.
}

completa de la versión poética. En efecto, si es cierto que «conforme van avanzado las prosificaciones, se observa una condensación evidente de los contenidos de las cantigas originales. A veces, incluso, se eliminan detalles y la narración se hace cada vez más rápida y breve» ${ }^{68}$, no podemos pasar por alto que en ocasiones, sobre todo en lo que concierne el comportamiento de los judíos y su cohabitación con los cristianos, las prosificaciones aportan detalles altamente significativos, que podrían explicar tanto el espíritu con que fueron redactadas como el público al que se destinaban.

Sin llegar a ser auténticas glosas, las prosificaciones aportan una explicación y desarrollo, casi un comentario, de la versión poética del milagro. Por ello, podemos empezar a comprender que el lector al que se destinaban las prosificaciones no debía tener los mismos referentes culturales que el auditorio de las Cantigas en verso. Las narraciones en prosa serían, las explicaciones de intelectuales destinadas a un público menos culto, lo que a su vez nos deja ya intuir que estas prosificaciones podían destinarse a una auténtica lectura libresca, dirigida por un magíster ante un público ${ }^{69}$ con el que mantiene cierta relación de docencia $^{70}$.

${ }^{68}$ Ibid., p.37.

${ }^{69} \mathrm{Ibid} .$, p.36: «las prosificaciones no son en modo alguno resúmenes o simples traducciones literales (aportan nuevos elementos, más desarrollados). (...) Las prosificaciones se llevaron a cabo, seguramente, para que las gentes, que no conocían el gallego, pudieran entender los milagros, sobre todo los narrativos, ya que son los más interesantes desde el punto de vista moral o didáctico».

70 S. REYNOLDS, Medieval reading, Cambridge, Cambridge University Press, 1996, p. 28-29: «The kind of reader who would need the information embodied in the glosses, that is to say, grammatical information, is by definition the kind of reader who cannot write: someone who could write would, in terms of the twelfth-century hierarchy literate skills, have no need of such glosses. The only way out of this paradox is to 
Pero al estar inscritas en el propio códice destinado a la capilla real, y por lo tanto a un ambiente relativamente cerrado, no parecen haber sido redactadas para un público iletrado, salvo si estas prosificaciones debían ser el objeto de una réplica o copia en masa, más acorde con los libros de tipo universitario de los que hablábamos antes, o por lo menos para libros destinados a una lectura en un lugar más o menos público, tal vez en los propios refectorios de los monasterios ${ }^{71} \mathrm{o}$ como manual para el aprendizaje de la lectura para damas nobles o incluso retoños de los comerciantes adinerados.

Ello podría explicar las alteraciones narrativas que, con respecto a las Cantigas, se encuentran en las prosificaciones, sobre todo en la 15 y la 22.

Los primeros elementos suplementarios corresponden a lo cotidiano, aportan un sabor a verdad que enriquece en cierta forma el conjunto de la narración. Así, en la versión rimada de la cantiga 15 , el trovador confiesa ignorar el nombre del emperador,

see glosses as the written vestiges of a reading undertaken by experts for those who are not experts, that is to say, as a reading by a teacher for his pupils. Rather tan an individual, we have a generic, professional reader; the teacher or grammaticus who reads for others. This means in turn that even though the reading is not actively undertaken by the pueri, its nature is determined by them, for the mode of reading is directly dependent on the level of their literacy. While they do not read (in our sense of the term) the text at all, for it remains at all moments and in all senses in the teacher's hands, their needs determine the use to which the text is put. This a communal reading, communicated orally $(\ldots)$ the glosses are merely the written traces of a much fuller reading practice, and what is more, they are part of a shift in the history of reading itself, away from the solitary rumination of monastic lectio to the more public forum of the classroom».

${ }^{71}$ O. PÄCHT, op. cit., p. 35 : «Il faut très probablement imputer ce changement aux mouvements de réforme des différents ordres monastiques, qui accordaient une valeur nouvelle entre autres, au respect des textes et préconisaient souvent la lecture de la Bible au réfectoire». marido de la emperatriz Beatriz, mientras que en la versión en prosa el emperador es identificado con Aurelio. No es que ese nombre indique la veracidad de los hechos ni permita situar temporalmente la historia, pero la transforma en algo plausible ${ }^{72}$, sobre todo para el pueblo llano. De la misma forma, la prosificación 22 nos indica que el personaje central, un labrador, se llama Mateo y que cultiva mijo; son datos de la vida cotidiana, próxima de los lectores, haciendo más palpable el milagro al inscribirlo en un mundo que les es conocido, o que puede formar parte de un referente más universal, como podría serlo el contenido en el ciclo artúrico.

Son dos las prosificaciones que pueden justamente inscribirse en este universo. La primera es sin duda la correspondiente a la cantiga 23. El rey de Bretaña de paso por sus posesiones tiene que escoger posada en casa de una noble dama que no posee suficiente vino para acoger a su señor; en respuesta a su súplica, la Virgen provee la bodega de la dama con un vino de origen celestial. La prosificación se inscribe más en el contexto artúrico, puesto que aporta una mayor descripción de la reacción del monarca y de su séquito ante el vino propuesto por la dama bretona. Esta versión insiste en la presencia del rey y de sus caballeros, que ensalzan la excelencia del vino propuesto, cuando en la cantiga original sólo ocupan un papel segundario. Esta situación, que apenas queda evocada en los versos, podría ser el reflejo, más o menos directo, del estereotipo del monarca artúrico, en compañía de bravos compañeros, recorriendo el país, recordando y comentando sus aventuras.

${ }^{72}$ F. MUNDI y A. SAíz, op. cit., p.46: «la prosificación pretende ser más histórica que la cantiga gallega o, por lo menos, asentarse sobre unas bases aparentemente reales y verídicas» 
Siempre dentro de la categoría más o menos conforme a la leyenda artúrica y de las aventuras de caballería se sitúa el poema 19 y sus tres caballeros exiliados por el asesinato de un rival ante el altar de la Virgen. La cantiga explica el origen del acto sacrílego, realizado "por prazer do demo, que os seus aguilla» (v.13) pero la redención final queda mucho más desarrollada en la prosificación, puesto que es el último caballero quien cuenta su historia al pueblo mostrando, como prueba de lo acaecido, el silicio que lleva incrustado en las carnes. Se retoma así la imagen del caballero pecador, transformado en ermita que sufre por sus pecados e intenta educar a los demás con sus desdichas, rebasando con creces la imagen del caballero en el exilio presentado por la versión en verso y que no destaca las consecuencias del castigo divino, poniendo sobre todo el acento en la ejecución de la justicia divina.

Forzoso es reconocer que las informaciones suplementarias no rebasan el marco de lo puramente anecdótico, y son muy superficiales cuando se refieren a acontecimientos históricos supuestamente demostrados. A lo sumo, permiten inscribir la narración dentro de una tradición literaria que enmarca tanto el aspecto religioso como el caballeresco. El deseo de añadir elementos que den una impresión de veracidad, y que se inscriben dentro de una tradición literaria más clásica, podría indicar la necesidad, por parte del público, de abandonar la estructura abstracta para adentrarse en un mundo lleno de referentes conocidos o familiares.

La diferencia entre ambas percepciones es importantísima puesto que en lo que se refiere a los judíos, las prosificaciones no hacen sino subrayar los estereotipos, aban- donando los matices que podemos encontrar en las versiones rimadas ${ }^{73}$.

La cantiga número 3, que narra la historia de Teófilo, nos recuerda que el personaje central «per consello dun judeu por gãar poer» (v19-20) entró en contacto con el diablo, a quien le vendió su alma. En esta cantiga la relación entre el judío y el demonio se da por sentada, aunque no se presenta en ningún momento una relación de vasallaje directo del primero hacia el segundo y desde luego no se recalca el mito del judío como hechicero. Muy diferente es la versión en prosa según la cual el judío era un brujo vasallo de satanás y que intentó embaucar a la esposa de Teofilo por considerarla más débil en su fe. Sería ésta quien causaría, en teoría, la pérdida del marido, aunque le judío no supo calcular la fuerza de la devoción religiosa de la espo$\mathrm{sa}^{74}$ que supo vencer las acometidas del demonio.

Nos encontramos aquí con dos aspectos sumamente importantes: la hechice-

\footnotetext{
${ }^{73}$ La narración en prosa del milagro de san Ildefonso, recuerda que "como las gentes eran nuevas en la creençia de la santa fe catholica, avía entonces muchos erejes que dubdavan en la virginitat de Santa María e en otros artículos de la fe (...) Sant Alifonso com alunbbrado de Espíritu Santo que ordenó muchos libros (...) contra la ceguedat de los erejes e contra la porfía de los judíos». La idea de novedad del catolicismo en la península evoca sin duda la transición del arrianismo hacia el catolicismo, al par que refuerza la idea de antisemitismo, muchas veces matizada y atenuada en la cantigas en gallego, que recuerdan las alabanzas que hizo el santo «da virgiidade daquesta Señor miuy santa, per que sa loor tornada foi en Espanna de quanta a end' avian deytada judeus e a eregia» (ctg 2, v21-25).

74 «A los tales tienpos, que veno (el diablo) a fablar con un judío encantador quel tenía por su siervo tienpo avía (...) E el judío encantador, pora la carga que del diablo teníe, púsolo luego en obra, e entendiendo que a la muger que la fallaríe más flaca, a la mover a esta razón, e que ella después que moveríe a su marido, veno primero a ella e ella, commo servidora de Santa María e nunca perdiendo della fiuza, dixo quella nunca tal negocio faría»
} 
ría atribuida al judío, y su consecuente sumisión al demonio, y la debilidad de la mujer, habitualmente asociada al personaje de Eva y al pecado, salvo cuando se acerca a María ${ }^{75}$. Lo más interesante es que esta versión en prosa se acerca sobremanera de la versión de Berceo e incluso de Coincy para quienes el judío es el vasallo y servidor del demonio, aunque el papel de la mujer queda mucho más difuso en sus versiones.

Las prosificaciones transmiten por lo tanto una visión estereotipada del judío, una visión que está ausente de las versiones poéticas destinadas, según nuestra hipótesis, a un público mucho más culto que seguramente no defendía semejantes tesis. Esta idea se podría confirmar con la cantiga 6. Esta narración aporta una descripción social nada desdeñable puesto que se refiere a una fiesta en la que los cristianos y los judíos cohabitan y se divierten juntos, tal y como se puede observar en la segunda miniatura. Un muchacho canta entonces una cantiga en honor de la Virgen y su canto era tan maravilloso que «foron en mui pagados todos, senon un judeu que lle quis gran mal des ende» (v.40). En otras palabras, la versión en verso deja entender que el canto no ofendió a ningún judío salvo uno, y ello a pesar del tema abordado. La prosa presenta otro cuadro, puesto que aquí «este moço cantó su cantar; e a los cristianos plazielos e los judíos avían dende grant pesar. E un judío de los que jugavan tobo ojo a este moço para se vengar dél». El marco es el mismo, pero la prosa insiste en una separación cultural que no existe ni en la versificación ni en la miniatura correspondiente. Por ello, si parece evidente que «estas prosificaciones irían dirigidas a aquellos castellano-parlantes que querían entender las

\footnotetext{
${ }^{75}$ femina provendría de fe minus, la que tiene menos fe.
}

Cantigas al leerlas o escucharlas» ${ }^{76}$, también debemos considerar la posibilidad de que se destinaran a un público que sólo atiende a aspectos clásicos y estereotipados, en un momento en el que la armonía entre culturas que podía existir en la corte alfonsí bien podría haber sido alterada. Recordemos en este sentido que en 1280 el monarca castellano hizo ejecutar a su almojarife, Zag dela Maleha, por haber dado el dinero destinado a la contienda de Algeciras al heredero, Don Sancho; éste lo utilizó para pagar el retorno de su madre, huida al reino de Aragón ${ }^{77}$. Ese mismo año Alfonso X impone un rescate a la comunidad judía del reino para reaprovisionar sus arcas.

Podemos adelantar la suposición que en el momento de redactar las prosificaciones, las relaciones con la comunidad judía, por parte del monarca ya gravemente enfermo, no disfrutaban de la misma armonía que en el pasado. Dicha degradación se reflejó en la descripción que de los hebreos se hace en la versión castellana, una descripción mucho más agresiva, semejante a la que nos propone, por ejemplo, Gauthier de Coincy ${ }^{78}$.

En resumidas cuentas, podemos aceptar que, mientras los trovadores y su público podían contentarse con contextos poéticos abstractos, apenas esbozados pero de los que sabían extraer el verdadero mensaje espiritual, para el auditorio de la versión castellana dicho mensaje sólo podía ser considerado auténtico si el contexto parecía verosímil refiriéndose a aspectos de la vida cotidiana o a estereotipos de lo más comunes. Ello parece confirmar que el público de las prosificaciones no sólo no comprendía el

\footnotetext{
${ }^{76}$ F. MUNDI Y A.SAÍZ, op. cit., p. 45 .

${ }^{77}$ H. SALVADOR MARTÍNEZ, p. 444 y siguientes.

${ }^{78}$ G. DE COINCY, op. cit., p. 82: «L'incarnation Jhésucrist longtens nous veulent dénoier,. On les devroit pendre ou noier. Li déables leur dort es testes qui bestiaux les fait com destes».
} 
gallego-portugués, sino que tampoco podía juzgar la veracidad de los elementos narrados.

Ahora bien, el impacto de las versiones castellanas sobre el auditorio sólo puede explicarse con relación a la existencia de una obra superior y más perfecta, aunque nunca percibida ${ }^{79}$.

Todas las prosificaciones terminan por una frase recordatoria que, con alguna que otra variante, se puede resumir con la frase de la sexta prosificación: «e por ende el Rey don Alfonso, a loor de la Virgen, fizo la cantiga suso dicha», siguiendo después el estribillo correspondiente a la cantiga de referencia.

Estas frases relegan las prosificaciones a un segundo rango con respecto a sus respectivas versiones en verso; casi podríamos decir que las narraciones en prosa se presentan como un producto derivado de las realizadas en verso, y que por lo tanto sólo tienen cierto valor por ser el reflejo de una obra mayor y más acabada. Esta segunda versión de los milagros bien podría por lo tanto no haber sido del todo dirigida por el monarca castellano, y representar una creación mucho menos personal que las cantigas. Todo ello nos lleva a comprender que el hipotético lector de las prosificaciones tendría siempre en mente la existencia de una obra superior, más perfec-

\footnotetext{
${ }^{79}$ H. MARTIN, op. cit., p. 100 : «La pédagogie chrétienne est analysée en termes limpides: un discoursamont commande pour l'essentiel le programme iconographique; une réception-aval par les fidèles entraîne un effet de ressac sur cette même iconographie. L'examen des textes suggère forment que les images, les sermons et le théâtre constituaient les éléments inséparables d'un seul et même enseignement religieux». P. 115 : «Les phénomènes de cascade hiérarchique sont fréquents, dans le domaine politique ou religieux. Exemple type : un ange transmet le message divin à un prophète, qui le répercute au peuple. La transmission elle-même est figurée concrètement par un phylactère».
}

ta, que quedaba fuera de su alcance y que se encontraría en la capilla real.

En cierto modo, se va estableciendo así la idea de la presencia de una verdad superior, inalterable, a veces fuera del alcance del común de los mortales. Así, pese a la hipotética divulgación de las Cantigas, tanto en su versión cantada por los trovadores como en su versión en prosa leída por algún magíster, el manuscrito de las CSM se transforma en una reliquia ${ }^{80}$, única e inigualable, que inspira al hombre y que se encuentra cerca del monarca, en una especie de sancta santorum, como podría estarlo el Arca de la Alianza en el templo de Salomón.

Pese al desarrollo universitario del siglo XIII, y la autonomía que va tomando paulatinamente el mundo intelectual con respecto a la Iglesia, la posesión del libro sigue expresando una distinción social significativa. No es tanto el contenido mismo del libro lo que parece diferenciar los diferentes tipos de dueños sino la factura del mismo. La riqueza de su composición sigue siendo una marca de la nobleza o de riqueza del que lo posee, pero la forma en que está redactado demuestra también un tipo diferente de lector.

El siglo XIII, sobre todo bajo el rey sabio, es un momento de auge intelectual en el que el analfabetismo parece ir disminuyendo, gracias a la multiplicación de los temas abordados en los centros de producción y docencia. Sin embargo, pese a la

\footnotetext{
${ }^{80}$ La cantiga 209 nos narra cómo, estando el rey en Vitoria, padeció una enfermedad de tal intensidad que los médicos fueron incapaces de encontrar un remedio o aliviar el dolor del monarca. El rey pidió entonces que le trajeran el manuscrito de las Cantigas, al contacto del cual sanó inmediatamente. El manuscrito permitió así la realización de un milagro y dejó de ser una colección de milagros, como todas las demás, para convertirse en un medio por el cual se manifestaba el poder de María y de Dios.
} 
evolución temática y la proliferación más o menos importante de volúmenes escritos, la lectura medieval del siglo XIII, sobre todo en lo que concierne la narración de tipo religioso o incluso historiográfico, sigue construyéndose sobre tres pilares esenciales: vidi, audivi, legi ${ }^{81}$. El vidi sigue siendo la prueba material de la veracidad de lo narrado. Por una parte, el autor de una narración milagrosa expresa así la autenticidad de lo narrado por proponer su propia persona y reputación como garantía de veracidad, paralelamente, el lector, o mejor dicho espectador, que observa la historia bajo forma de representación iconográfica (miniatura, pintura, escultura $)^{82}$ no puede tener dudas sobre la posible realidad de lo narra$\mathrm{do}^{83}$. Este tipo de transmisión de la narración que en cierto modo unifica el mensaje y evita interpretaciones y representaciones aleatorias queda reforzado por el audivi. Por supuesto, una historia que se ha oído contar podría ser alterada o exagerada, pero no

${ }^{81}$ S. MULA, «Les modèles d'autorité religieuse dans la narration profane (XIIe-XIIIe siècles)» in Auctor et Auctoritas, invention et conformisme dans l'écriture médiévale, Paris, 2001, p. 163-164 : «Vidi : c'est la source la plus fiable. L'auteur se pose comme autorité, sa bonne foi ne peut être mise en doute. Legi : les sources écrites, dans un monde qui se rappelait l'affirmation de saint Paul dans son Épître aux Romains avaient une valeur indiscutable. Audivi: dans un récit historique l'ouï-dire devrait être le point faible; Cependant si un homme qui s'impose par son ancienneté et sa religiosité raconte une histoire merveilleuse, on ne met pas en doute son témoigne».

${ }^{82}$ H. MARTIN, op. cit., p. 100: « les images parlaient abondamment, par les inscriptions qu'elles comportaient, et pouvaient être agencées comme des scènes de mystères. (...) les ymagines, ce terme désignant aussi bien les sculptures que les icônes, détenaient un pouvoir coercitif qui renforçait leur vertu pédagogique initiale».

${ }^{83}$ Ibid., p. 99: «les images faciliteraient donc la prière des fidèles en les dotant $\mathrm{d}^{\prime}$ un substitut vraisemblable de monde céleste. Mais la médaille comporte son revers, les représentations figurées pouvant avoir un pouvoir maléfique, en incitant au péché de la chair. Il faut donc mettre le pouvoir des images, pleinement reconnu, au service de la bonne cause, ce qui semble être devenu très courant à la fin du Moyen Âge». hay que olvidar que el cristianismo se ha construido sobre la evangelización es decir la transmisión oral de la palabra. El discurso oral es la piedra angular de la transmisión del conocimiento, cimentada por la personalidad incuestionable del orador que reviste el papel de auctoritas. La debilidad de la palabra transmitida oralmente es que se encuentra rápidamente a la merced de la interpretación del auditorio, puede padecer alteraciones, incomprensiones y olvidos, de ahí la necesidad de aprender de memoria, de repetir siempre los mismos textos, ya sea en el ámbito religioso o en el puramente docente. Y lo más importante es que esta debilidad no desaparece con la utilización de la escritura. La palabra escrita y por lo tanto leída, el legi, debería ser el último peldaño de la obtención de la información.

Sin embargo, como se ve con las $C S M$, la lectura medieval aún no se conforma del todo con nuestra propia concepción de la lectura. Se lee en voz alta para un público, que no es que no sepa del todo leer, sino que manifiesta que la acción cristiana se hace en común, el acto solitario parece ser rechazado y de hecho la lectura personal e individual no es en líneas generales silenciosa, sino murmurada.

Por supuesto, y siempre basándonos en el ejemplo que nos proporcionan las $C S M$, observamos que la lectura no se realiza únicamente sobre un texto escrito ni tiene como límites la enunciación monótona, en su sentido más estricto de la palabra, del enunciado. $Y$ es que la lectura se compone tanto de una faceta oral como de otra escrita y visual, pero todas pueden seguir teniendo un punto en común, no sólo por el tema tratado, sino también por la relación que mantienen con la noción de belleza, entendida como una manifestación de Dios. 